\title{
EBL2, a flexible, controlled EUV exposure and surface analysis facility
}

\author{
Edwin te Sligte, Norbert Koster, Freek Molkenboer, Alex Deutz \\ TNO, Stieltjesweg 1, 2628 CK Delft, The Netherlands
}

\begin{abstract}
TNO is building EBL2 as a publicly accessible test facility for EUV lithography related development of photomasks, pellicles, optics, and other components. EBL2 will consist of a Beam Line, an XPS system, and sample handling infrastructure. EBL2 will accept a wide range of sample sizes, including EUV masks with or without pellicles. All types of samples will be loaded using a standard dual pod interface. EUV masks returned from EBL2 will retain their NXE compatibility. The Beam Line provides high intensity EUV irradiation from a Sn-fueled EUV source. EUV intensity, pupil, spectrum, and repetition rate are all adjustable. In-situ measurements by ellipsometry will enable real time monitoring of the sample condition. The XPS will be capable of analyzing the full surface area of EUV masks and pellicles, as well as performing angle resolved analysis on smaller samples. Sample transfer between the XPS and the Beam Line will be possible without breaking vacuum.
\end{abstract}

Keywords: EUV exposure mask pellicle XPS analysis metrology handling

\section{INTRODUCTION}

EUV is approaching its insertion point for high volume manufacturing as EUV sources and EUV lithography tools become increasingly mature products. This entails a need for development of both EUV mask and pellicle infrastructure, as well as continuing the EUV scanner product and source power roadmap. Many issues remain to be addressed in these fields, and testing will play an indispensable part in resolving these issues.

TNO International Centre for Contamination Control (ICCC) is dedicated to developing the highest standards and practices in contamination control, for preventing and eliminating both particle contamination and molecular contamination. In 2005, TNO and Carl Zeiss SMT GmbH jointly established the unique EBL test facility to further the development of EUV technology. EBL contains an EUV Beam Line, in which samples can be exposed to EUV irradiation in a controlled environment. Attached to the Beam Line is an XPS system, which can be reached via an in-vacuum sample transfer system. This enables surface analysis of exposed samples without breaking vacuum. The compound instrument is used to develop and validate optics lifetime strategies for ASML EUV scanners ${ }^{1}$.

EBL was not designed for exposures of EUV masks, pellicles, and the intensities currently foreseen in EUV product roadmaps. As it became clear that EBL was no longer fully satisfying industry demand, and the need for a publicly accessible EUV exposure facility grew, TNO decided to invest in a successor system, called EBL2. The concept study was described in Ref.[2]. The present paper gives an overview of the EBL 2 system design, and provides more detailed information on accepted samples, the sample handling system and XPS modules.

During the design process, TNO entered into two strategic technology partnerships with companies that share TNO's vision to speed up the development of next-generation lithography systems, masks, and pellicles. Ushio Inc. is the technology partner in the EUV source, and ASYS Automatic Systems GmbH \& Co. KG contributes its mask handling technology.

Photomask Japan 2016: XXIII Symposium on Photomask and Next-Generation Lithography

Mask Technology, edited by Nobuyuki Yoshioka, Proc. of SPIE Vol. 9984, 99840R

(C) 2016 SPIE · CCC code: 0277-786X/16/\$18 - doi: 10.1117/12.2240302

Proc. of SPIE Vol. $998499840 R-1$ 


\section{SYSTEM OVERVIEW}

An overview drawing of the EBL2 system is shown in Figure 1 below. The main system components are an EUV source (labelled a), a Collector module (b), an Exposure chamber (c), a sample handling system (d), and an XPS analysis system (e).

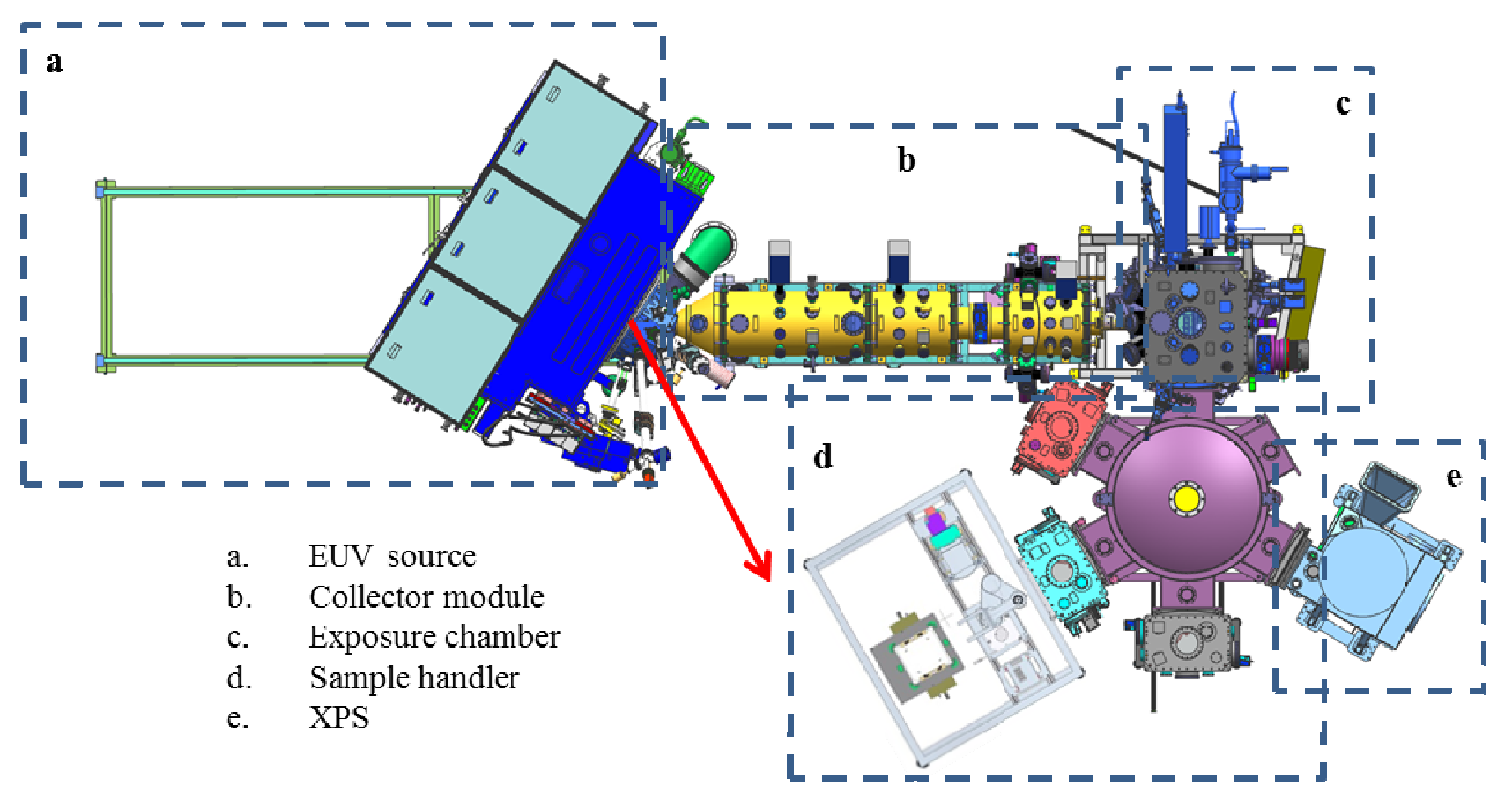

Figure 1: EBL2 CAD design overview. The arrow indicates the beam path out of the Metrology Output Port

The USE-3315E Sn-fueled LDP EUV source is provided by Ushio. Details on the operating principle can be found in Ref. [3]. It provides high power pulsed EUV light to the beam line, with a pulse repetition rate of 3 $\mathrm{kHz}$, that can be tuned up to $10 \mathrm{kHz}$ on request. For low-power metrology applications, a highly flexible metrology output port is available at the EUV source. The horizontal beam path through this KF40 port is indicated by a red arrow in the schematic drawing.

The Collector module projects the high power EUV from the source into the Exposure Chamber, while decoupling the two vacuum environments. The collector optics have an intermediate focus to enhance the differential pumping performance. The in-band EUV intensity reached at the sample will exceed $1 \mathrm{~W} / \mathrm{mm}^{2}$ in focus. The Collector Module will enable insertion of filters in a separate vacuum chamber to modify the spectrum, pupil, or both. In focus, the EUV spot will have a typical FWHM of $1 \mathrm{~mm}$. Larger spot sizes up to $30 \mathrm{~mm}$ diameter can be achieved using a defocusing mechanism, which moves the entire Collector module and EUV source composite.

Samples are exposed to EUV in a flexible and controlled manner in the Exposure Chamber. The sample (mask or other; see below for details) can be loaded on a chuck by automated hardware. The chuck is temperature controlled and contains sensors for measurements of both EUV power and spot profile. The gas environment is very flexible, enabling high vacuum exposures $\left(<10^{-6} \mathrm{mbar}\right)$ and exposures in a high purity purge gas $\left(\mathrm{H}_{2}, \mathrm{He}\right.$, Ar) up to 4 mbar, with added contaminants $\left(\mathrm{H}_{2} \mathrm{O}\right.$, XCDA, or specialty contaminants). A differentially pumped RGA enables control of the gas composition. In-situ measurements of the optical properties of the sample by ellipsometry will enable real time monitoring of the sample condition. Also, the 
imaging ellipsometer will serve as an alignment tool, maintaining overlay between sequential EUV exposures and other process steps.

Various types of samples are accepted, as described in Section 3. ASYS provides the Sample Handling equipment, which consists of a vacuum handler and an atmospheric handler. The performance of the Handling Equipment is described in more detail in Section 4. XPS analysis on EUV masks and other samples is possible with the XPS system, described in Section 5.

\section{SAMPLE HOLDERS}

EBL2 will accept EUV masks with or without pellicles, as well as a wide variety of other samples, provided these samples meet the interface requirements of the sample handler. Figure 2 shows several sample holders available as standard.

Figure 2a shows a sample holder for small samples suited to both EUV exposure in the beam line and subsequent in-situ XPS analysis. The sample holder has two slots for 1 inch diameter, $1 / 4$ inch thick samples. Smaller samples can be accommodated by custom made adapters. The red planes in the sample holder are the interfaces for handling the sample holder. The red marks that can be seen at the bottom of the sample holder match the reticle inner pod base plate interface. Custom sample holders for different geometries are possible provided they fit in this volume $(190 \mathrm{~mm} * 152 \mathrm{~mm} * 20 \mathrm{~mm})$ and have the appropriate handling interfaces.

Reticles are accepted in SEMI standard dual pods ${ }^{4}$, and will be handled inside the EBL2 system on inner pod baseplates. Figure $2 \mathrm{~b}$ shows an exploded view of the dual pod assembly. From top to bottom, the components are the outer pod cover, inner pod cover, reticle on inner pod base plate, and outer pod base plate. Reticles with pellicles mounted easily fit in the envelope of the sample holder in Figure 2a, and so can be treated the same way as plain masks throughout the system.

A separate XPS sample holder will enable batch analysis of small samples in XPS. This sample holder can contain up to 61 inch samples, as shown in Figure 2c. This row of samples can be rotated by a mechanical actuator in the XPS stage for angle resolved measurements.
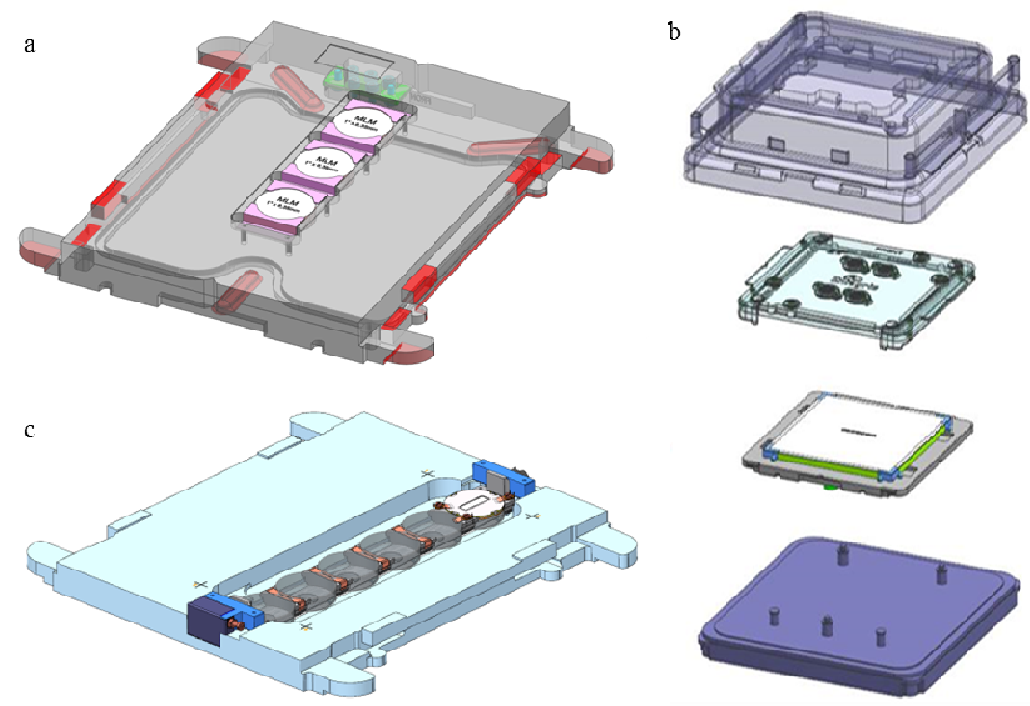

Figure 2: Types of sample holders used in EBL2. a) custom sample holder for exposure of small samples, b) EUV mask in dual pod, and c) XPS sample holder for small samples (courtesy of Kratos). 


\section{SAMPLE HANDLER}

The sample handler consists of two parts: an atmospheric handler (shown in the bottom left of area d in Figure 1 ), and a vacuum handler (the rest of the handler). Both are provided by technology partner ASYS. The automated sample handling infrastructure accepts SEMI standard dual pods, and maintains NXE compatibility for backside particle contamination. This need translates into a requirement that 600 loading cycles will result in addition of no more than 2 particles with a diameter of bigger than 10 micron, and no more than 48 particles with a diameter between 3 and 10 micron.

EBL2 will be located in an ISO 8 clean room; the atmospheric handler will provide a locally cleaner minienvironment to meet the particle cleanliness requirement. All sample holders are provided in a SEMI standard outer pod for generic interfacing; only the outer pod will come into contact with the ISO 8 environment.

Figure 3 shows the atmospheric and vacuum handlers. The atmospheric handler unloads the custom sample holder from the outer pod, after which it can be loaded into and unloaded from the load lock. For reticles, several options are available. Depending on user preference, reticles can be loaded onto a dedicated EBL2 inner pod base plate, or remain on their 'own' baseplate. A storage unit (shown right of the robot in Figure 3) provides the EBL2 inner pod base plate. A rotation and flip unit (left of the robot in the Figure) can rotate the reticle in 180 degree steps to orient the reticle appropriately for the planned experiment. When configured as desired, the reticle can be loaded into the vacuum system.
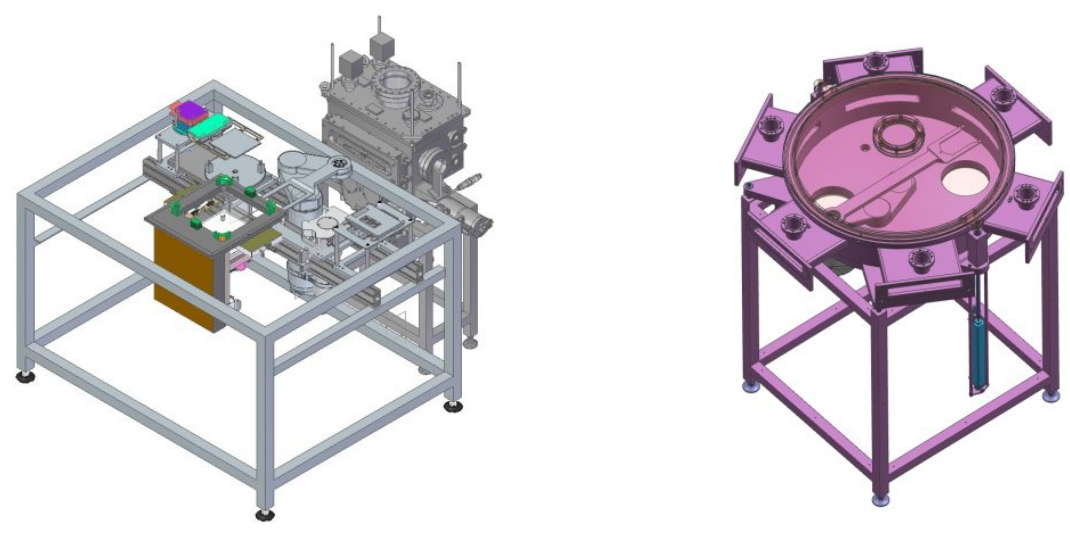

Figure 3: Left: atmospheric handler and load lock. Right: vacuum handler overview.

The sample holder is loaded into the load lock, where it rests on a kinematic mount. To ensure low particle addition during pump down and venting, laminar flows are required at all times. The load lock vacuum hardware has been designed to this specification using the particle transport modelling described in Ref. [5]. The vacuum handler robot can pick up the sample from the load lock once it is under vacuum. The vacuum handler robot lifts both sample holders and inner pod base plates at the red planes indicated at the bottom side of the sample holder in Figure 2a.

The vacuum handler is shown in the right hand part of Figure 3. It supports a base vacuum pressure of $10^{-8}$ mbar to provide a clean handling environment. The vacuum handling robot transfers the sample holder between the vacuum chambers attached to the vacuum handler. In a generic process, the robot moves under the sample holder and lifts it. It then retracts into the vacuum handler chamber, and rotates to face the destination chamber. When the valve to the original location has been closed, and that to the destination 
opened, the robot extends into the destination chamber, lowers the sample holder onto the destination mounts, and retracts into the vacuum handler chamber.

Attached to the vacuum handler are two storage chambers - one short term parking unit with in-vacuo cleaning functionality, and a long term storage chamber. The other chambers are the Exposure Chamber and the XPS. Each chamber is normally closed by a valve to prevent cross contamination between the system modules.

\section{XPS SYSTEM}

Surface analysis in EBL2 is possible through an XPS system, capable of analyzing elemental composition of the top several nm of a sample 6 . EBL2 contains a customized Kratos Axis Nova XPS system, shown in Figure 4. It will accept all samples handled by the Sample Handler.
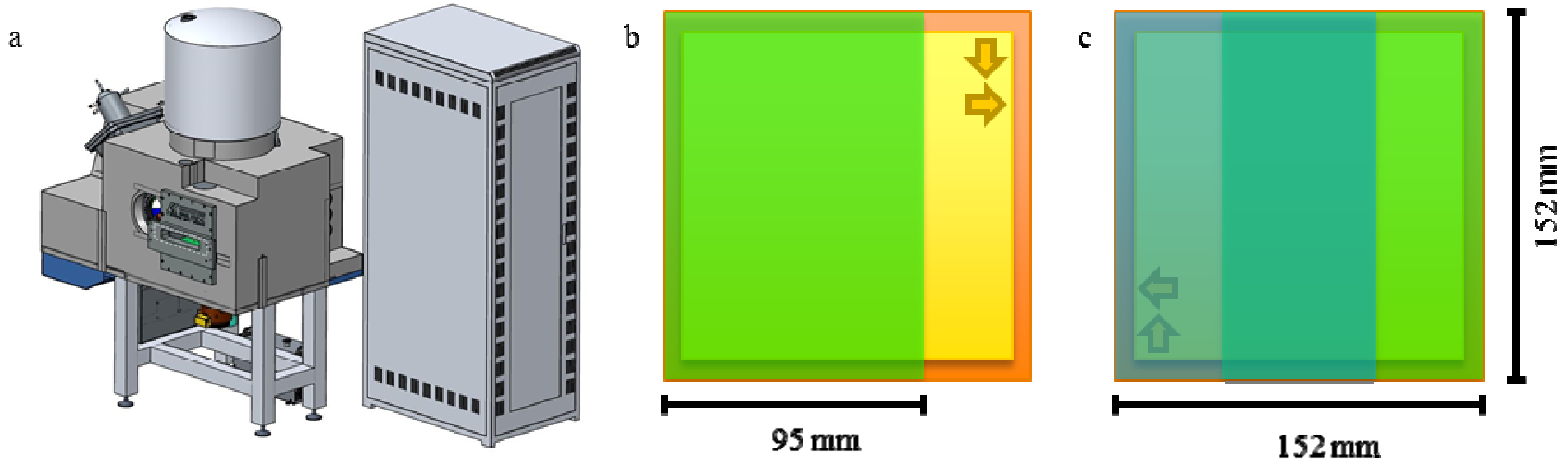

Figure 4: a) CAD model of EBL2 XPS(courtesy of Kratos); b) single scan accessible area on an EUV mask; c) accessible area with rotation.

XPS will be done using a monochromated $\mathrm{Al} \mathrm{Ka}$ X-ray source. The electron energy filter has a resolution of $0.1 \mathrm{eV}$ to enable detection of chemical binding states as well as elemental composition. The standard measurement spot size is $0.7 \times 0.3 \mathrm{~mm}$. Smaller spot sizes can be achieved at the expense of longer acquisition times through the use of apertures.

EUV masks will be analyzed on their base plates, avoiding physical contact with the XPS system. The XPS has an X,Y stage allows it to access $62 \%$ of the mask surface in a single scan, as shown in Figure 4b. The remainder of the mask area can be accessed after rotation in the atmospheric handler, as indicated in Figure 4c. Specific locations of interest can be defined, and the system can be instructed to examine only those locations. This enables defect mapping.

For small samples, the rotation actuator will enable angle resolved XPS analysis for enhanced surface sensitivity. The mechanical actuator will be able to rotate the row of small samples in the XPS sample holder up to 80 degrees. The mechanism will not affect EUV masks or inner pod base plates.

\section{CONCLUSION}

The EBL2 detailed design has been completed. Compared to the existing EBL system, large improvements in EUV power, intensity, metrology, reliability, and flexibility are achieved. 
All major components have been ordered, and system integration will take place in the second half of 2016. EBL2 will become accessible for users in the first quarter of 2017.

TNO would like thank its technology partners Ushio, Inc. and ASYS Automatic Systems GmbH \& Co. KG for the open and constructive collaboration in designing EBL2.

\section{REFERENCES}

[1] Noreen Harned, Roel Moors, Maarten van Kampen, Vadim Banine, Jeroen Huijbregtse, Roel Vanneer, Antoine Kempen, Dirk Ehm, Rogier Verberk, Edwin te Sligte, Arnold Storm, "Strategy for Minimizing EUV Optics Contamination During Exposure", EUVL Symposium, September 29 - October 2, 2008, Lake Tahoe

[2] Edwin te Sligte, Norbert Koster, Alex Deutz, Wilbert Staring, "A New Mask Exposure and Analysis Facility", in Proc. SPIE 9235, Photomask Technology 2014, 92351F (8 October 2014); doi: 10.1117/12.2083713

[3] Yusuke Teramoto, Bárbara Santos, Guido Mertens, Ralf Kops, Margarete Kops, Alexander von Wezyk, Hironobu Yabuta, Akihisa Nagano, Takahiro Shirai, Noritaka Ashizawa, Kiyotada Nakamura, Kunihiko Kasama, "High-radiance LDP source for mask-inspection application," in Extreme Ultraviolet (EUV) Lithography VI, Proc. of SPIE Vol. 9422 (SPIE, San Jose, CA, March 2015), pp. 94220F-1-9.

[4] SEMI E152-0214 - Mechanical Specification of EUV Pod for $150 \mathrm{~mm}$ EUVL Reticles

[5] Andreas Mack, Jacques van der Donck, Olaf Kievit, "Numerical Modeling of Particle Transport in Rarefied Flow", http://www2.avs.org/symposium2014/Papers/Paper_VT-TuM6.html

[6] John F Watts, John Wolstenholme, "An Introduction to Surface analysis by XPS and AES”, Wiley, 2003 